\title{
Research Paper \\ Preliminary Study of Psychometric Features of Wife Abuse Questionnaire
}

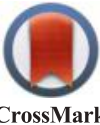

\section{Leili Panaghi ${ }^{1}$, Shahrbanou Ghahari ${ }^{2,3}$, Iraj Mokhtarnia ${ }^{4}$, Bita Dasarband ${ }^{5}$, Vahideh Nabavian ${ }^{5}$}

1. MD in Community Medicine, Associate Professor, Family Research Institute, Shahid Beheshti University, Tehran, Iran.

2. PhD in Clinical Psychology, Assistant Professor, Department of Mental Health, School of Behavioral Sciences and Mental Health (Tehran Institute of Psychiatry), Iran University of Medical Sciences, Tehran, Iran.

3. PhD in Clinical Psychology, Assistant Professor, Psychiatry and Behavioral Sciences Research Center, Addiction Institute, Mazandaran University of Medical Sciences, Sari, Iran.

4. PhD Student, Department of Basic Research, Family Research Institute, Shahid Beheshti University, Tehran, Iran

5. MA, Department of Families With Special Needs, Family Research Institute, Shahid Beheshti University, Tehran, Iran.

\begin{tabular}{|c|c|}
\hline & CFtation: Panaghi L, Ghahari Sh, Mokhtarnia I, Dasarband B, Nabavian V. [Preliminary Study of Psychometric Features of \\
\hline 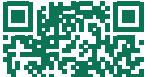 & $\begin{array}{l}\text { Wife Abuse Questionnaire (Persian)]. Iranian Journal of Psychiatry and Clinical Psychology. 2017; 23(2):218-231. https://doi. } \\
\text { org/10.29252/NIRP.IJPCP.23.2.218 }\end{array}$ \\
\hline 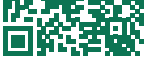 & doil": https://doi.org/10.29252/NIRP.IJPCP.23.2.218 \\
\hline
\end{tabular}

Received: 20 Jul. 2016

Accepted: 24 Jan. 2017

Key words:

Psychometric prop-

erties, Question-

naire wife abuse,

Women

\begin{abstract}
A B S T R A C T
Objectives The aim of this study was to examine the confirmatory factor structure and validation of wife abuse Questionnaire in sample women of subclinical.

Methods The study is cross-sectional and Married women's Mashhad city of formed the study population. Sample size of 671 women was selected by Availability sampling and the Qahari wife abuse Questionnaire with questionnaires Beck depression inventory, Sherer Self-Efficacy Scale and CTS-R questions given to was applied in the sample.

Results The results of confirmatory factor analysis support three factors. The Investigate Criterion validity by Pearson correlation coefficient in the wife abuse Questionnaire with The Beck depression inventory and Sherer Self-efficacy Scale, were between 0.31 and 0.45 and also convergent validity with Revised Conflict Tactics Scale, 60.0 correlation coefficient was obtained than are very satisfactory. A significant difference between the factor of questionnaire wife abuse in the women of divorce and non-divorce showed that the questionnaire has features of validity discrimination. The results of Cronbach's alpha coefficient in the Cronbach's alpha for the total questionnaire and factors were between 87.0 and 95.0 that was satisfactory.

Conclusion Therefore, based on the results of this study wife abuse's Questionnaire has psychometric properties applied for research and family counseling.
\end{abstract}

\section{Extended Abstract}

\section{Introduction}

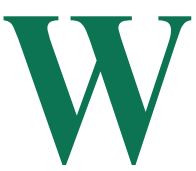

ife abuse is one of the most common phenomena in developing countries which leaves harmful effects on women's mental health and is considered one of the most important types of domestic violence, and is referred to the violence through which, men exercise their social or physical power on women. Based on research literature, wife abuse is divided into three general categories of abuse including: physical abuse, emotional abuse and sexual abuse. Given the importance of identifying, diagnosis, prevention and performing therapeutic interventions in the field of wife abuse, tools are needed to measure and assess wife abuse. In this regard, one of the Iranian made tools is the coercive spouse abuse questionnaire; although this questionnaire has been used in most Iranian studies, however, the psychomet-

* Corresponding Author:

Iraj Mokhtarnia, PhD Student

Address: Department of Basic Research, Family Research Institute, Shahid Beheshti University, Tehran, Iran.

Tel: +98 (914) 1907491

E-mail: mokhtarnia66@gmail.com 
ric properties of this questionnaire have not been comprehensively addressed in Iran. Therefore, the present study was conducted with the aim of evaluating the psychometric properties of the wife abuse questionnaire in a sample of women in non-clinical group.

\section{Method}

The method of the present study is an analysis of the correlation type. The statistical population of this study included all the married women of the city of Mashhad . By using available s ampling method, 671 subjects were selected among them which included two groups. The first group included 536 women who did not ask for divorce, and the second group included 141 women who were asking for divorce and had referred to the branches of family courts of the city of Mashhad. Sample inclusion criteria were those not asking for divorce; married, having passed at least one year of marital life, permanent marriage, and having at least completed the third-year of secondary school education. Exclusion criteria were: suffering from a mental disorder, and asking for divorce. Also among the inclusion criteria were asking for divorce, having passed more than one year of married life, permanent marriage, having filed petition for divorce and having completed at least the third year of secondary school education.

For data analysis the multivariate variance analysis test and Pearson correlation coefficient were used, and confirmatory factor analysis was through the diametric weighted least squares method, which were considered using SPSS21 and Lisrel 8.8 software. The error level of type one was 0.01 and the beta value was considered as 0.2 . Meanwhile all ethical principles including the principle of autonomy and the principle of confidentiality were observed in the research. The research tools were coercive spouse abuse questionnaire which was conducted on the sample group along with Sherer Self-efficacy Scale questionnaire and Beck's Depression Inventory, as the concurrent criterion validity, and conflict resolution tactics questionnaire as convergent validity.

\section{Results}

In the first stage, the Confirmatory Factor Analysis (CFA) method was used to investigate the unchangeability of spouse abuse questionnaire. Indicators obtained from factor analysis demonstrate that the index value of the square root of the mean squares of the approximate error was less than 0.08 which indicates the optimal fit and desirability of the model (RMSEA=0.031); also the square root value of the mean squares of the standardized remainder was 0.069 , in which the value less than 0.08 is considered as acceptable fit. Also, the indicator value of the goodness of fit of the model was 0.99 and the adaptive fit indicator of the model was 1 which indicates the acceptable fit between the threefactor model and the data. Also, the results of confirmatory factor analysis showed that all questions were identified in their own relevant factor and no question was deleted from the questionnaire.

For examining the concurrent validity, the questionnaire of conflict resolution tactics was used and for assessing the validity of the concurrent criterion, the tools of self-efficacy and depression were used, and the Pearson's correlation coefficients with spouse abuse questionnaire were respectively: $0.60,-0.40$, and 0.40 , which showed the convergent validity and concurrent criterion of the spouse abuse questionnaire were satisfactory. Also, in examining the validity of the questionnaire's structure, the correlation coefficients between spouse abuse questionnaire factors were studied and all correlations were significant at the level 0.01. The distinctive validity of spouse abuse questionnaire was performed by using multivariate variance analysis. And by comparing the mean scores of the two groups of married women asking for divorce and married women not asking for divorce, results showed that spouse abuse scores in women asking for divorce were higher than those who were not asking for divorce. In examining the reliability of the questionnaire the results of Cronbach's alpha coefficient in the total scale was 0.95 , in the emotional abuse factor was 0.93 , in physical abuse factor was 0.88 , and in sexual abuse was 0.87 , which were satisfactory.

\section{Discussion}

Based on the results obtained from this study, the coercive spouse abuse questionnaire has proper psychometric characteristics, with the explanation that the results of confirmatory factor analysis showed that the 3 factor model has desirably fit indicators; also all items of the questionnaire had acceptable load factor and were significantly uploaded on the desired factors. Resultantly, no items of any of the three factors: emotional abuse, physical abuse, and sexual abuse of the questionnaire were deleted. On the other hand, the self-efficacy and depression tools were used to examine the validity of the concurrent criterion in the individual performance dimension arising from spouse abuse.

Results showed that spouse abuse questionnaire has the capacity to anticipate the self-efficacy and depression of women. Therefore, the validity of this questionnaire criterion was also confirmed. In addition to having criterion validity for this questionnaire, the convergent validity was also confirmed and results showed that the spouse abuse 
questionnaire has also stronger connection with questionnaire similar to it.

It should be stated that reliability and validity coefficients suitable for this scale, short duration, ease of implementation and assessment of different dimensions of spouse abuse can make this questionnaire a suitable tool to be used for research purposes and for families' evaluation in family counseling, and It is also suggested to other researchers that, given the importance of this tool in research literature, they should validate these tools for applying them in clinical settings. On the other hand, as the content of this questionnaire assesses the personal and confidential issues of individuals' life, it is possible that the subjects act in a cautious manner to fill it out; this behavior can undermine the validity of the research, which can be one of the limitations of the present study.

\section{Acknowledgments}

This research did not receive any specific grant from funding agencies in the public, commercial, or not-forprofit sectors.

\section{Conflict of Interest}

The authors declared no conflicts of interest. 


\title{
بررسى مقدماتى ويثزتى هاى روانسنجى يرسش نامه همسرآزارى زنان
}

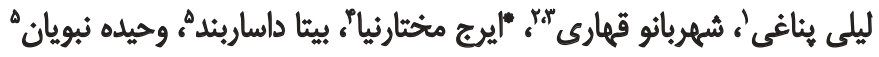

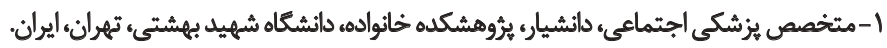

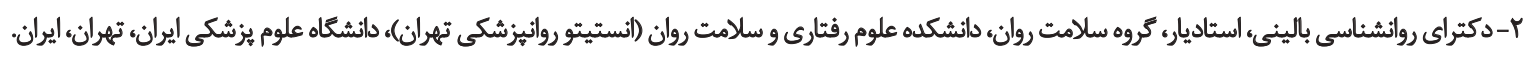

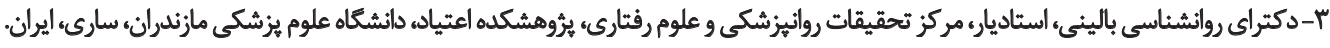

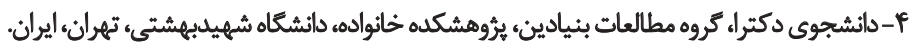

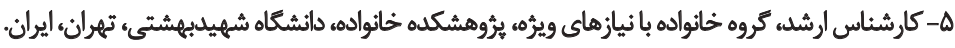

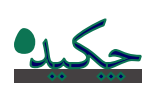

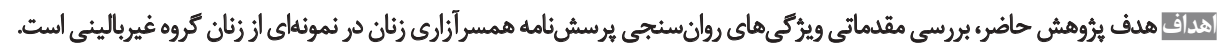

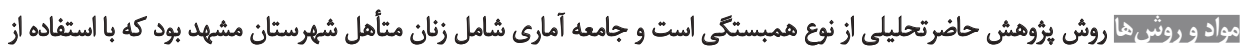

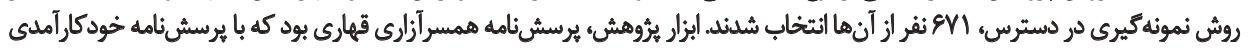

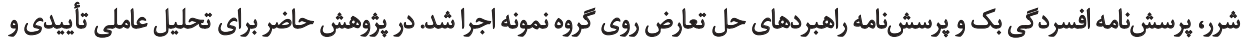

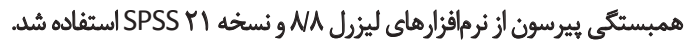

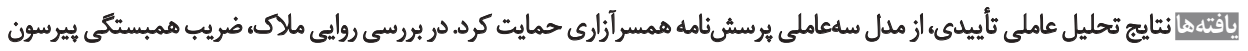

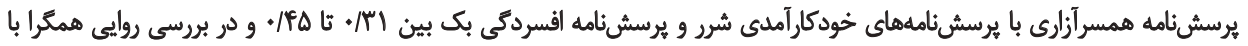

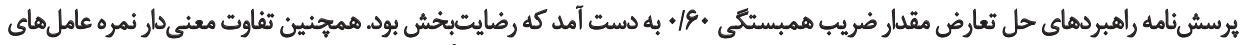

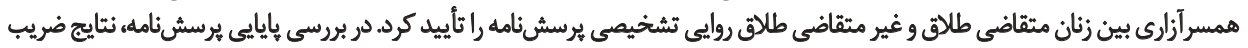

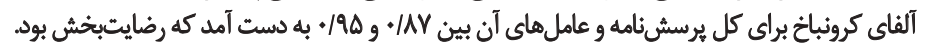

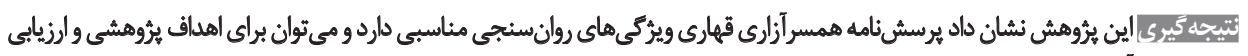

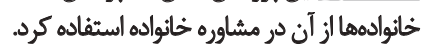

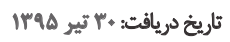

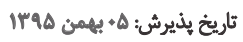

كليدوازٔهذا:

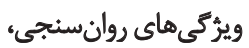

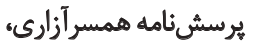
زنان

در بر مى كيرد. بر اساس دستهبندى ريجاردسون" و همكاران dotes

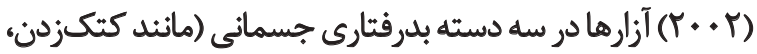

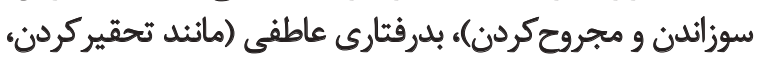

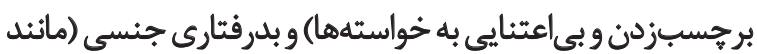

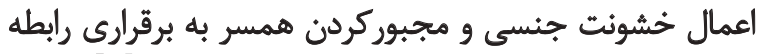

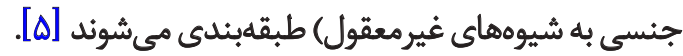

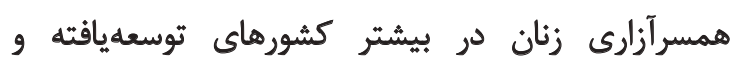

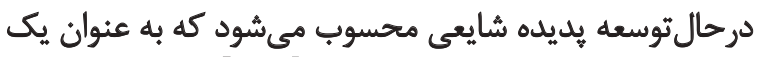

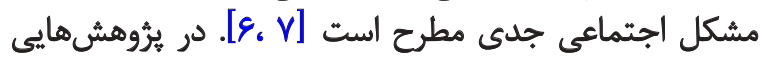

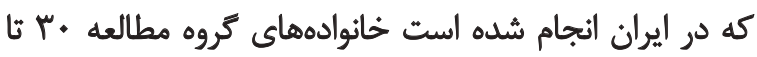

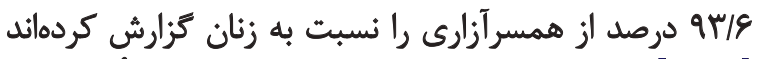

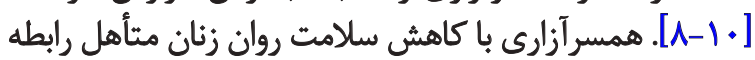

خشونت خانكى' مشكل شايع بهداشت عمومى در سراسر

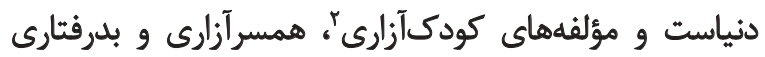

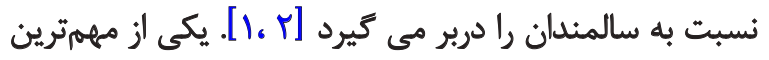

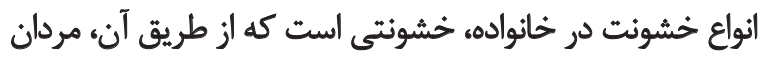

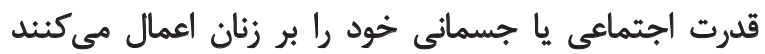

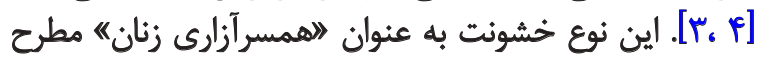

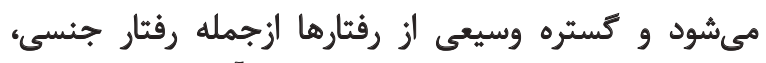

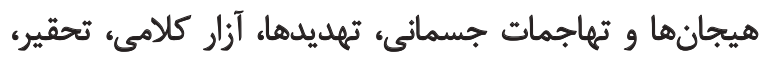

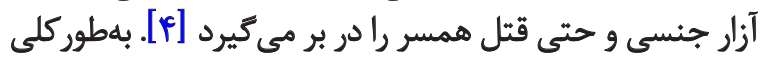

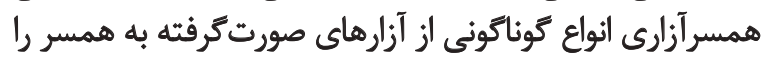


مثأهل شهرستان مشهد است. نمونه مدنظر از دو كروه تشكيل شد.

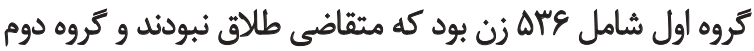

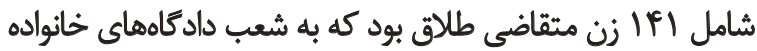

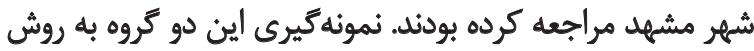

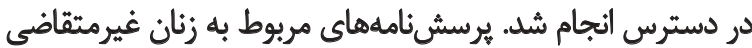

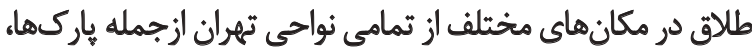

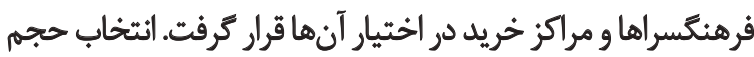

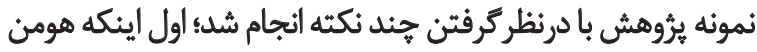

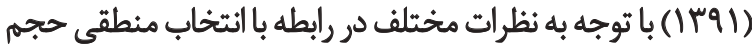

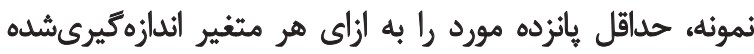

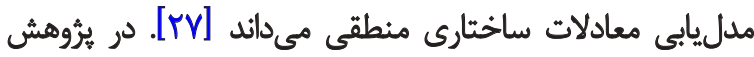

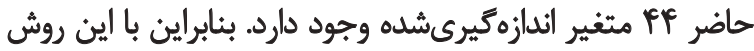

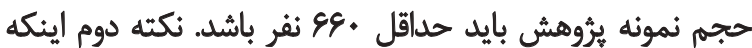

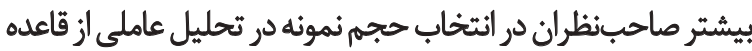

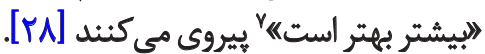
در اين يُروهش سعى شد از حجم نمونه بالايى استفاده شود.

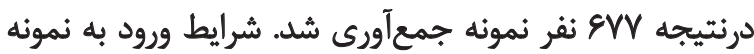

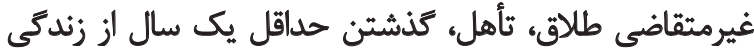

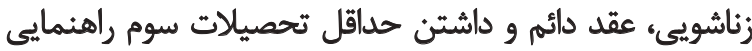

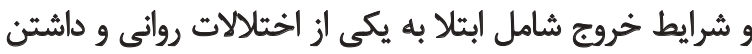

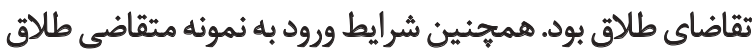

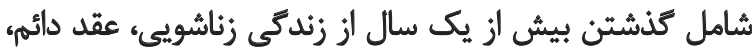

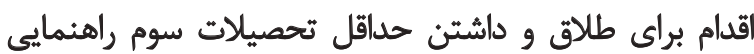

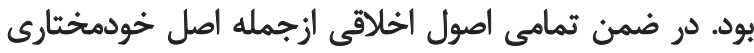

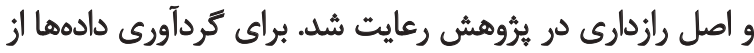
يرسشنامهاي زير استفاده شد:

$$
\text { يرسش نامه همسر آزارى }
$$

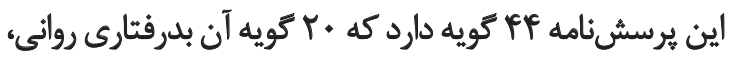

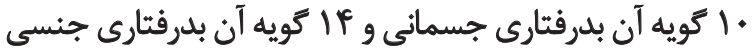

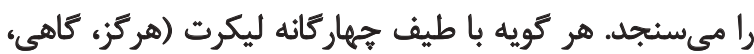

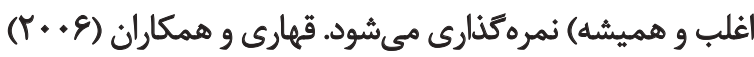

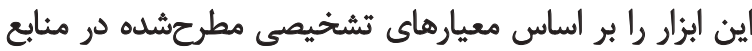

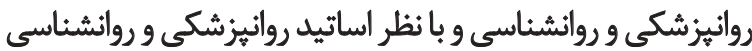

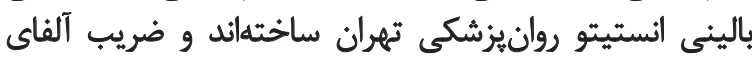

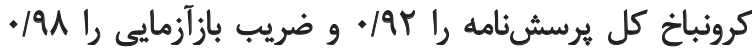

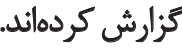

\section{نسخحه تجديدنظر شده هير سش ثامه راهبردهاى حل تعارض^^}

استراوس و همكاران (1999) اين يرسشنامه را به منظور

\section{More is better}

8. Conflict Tactics Scale-Revised (CTS-R)

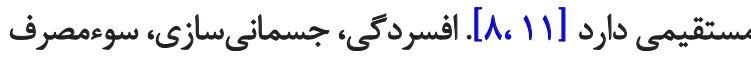

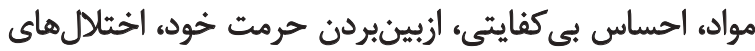

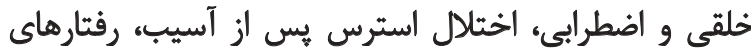

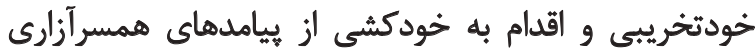

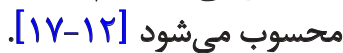

در بيشتر يروهشها به بيشكيرى از مشكلات ناشى از

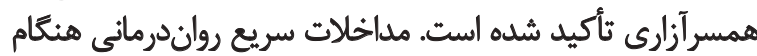

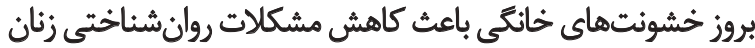

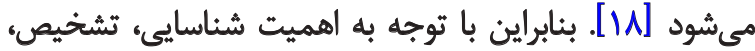

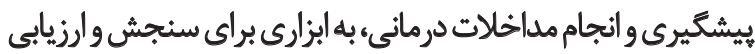

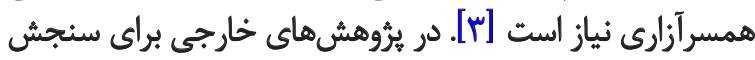

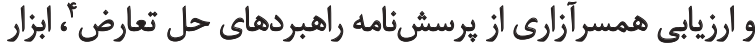

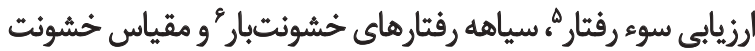

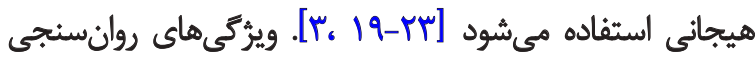

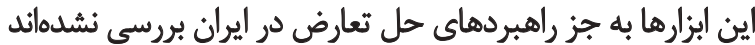

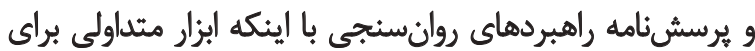

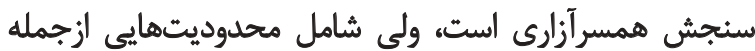

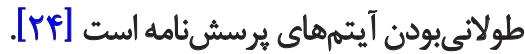
در ايران براي ارزيابى و سنجش همسرآزارى علاوه بر

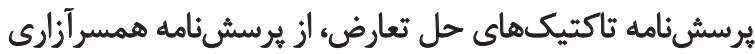

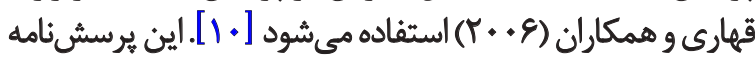

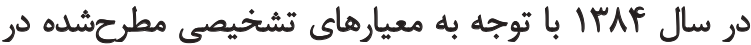

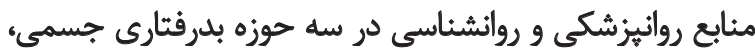

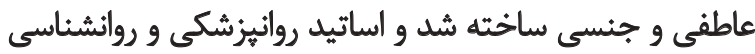

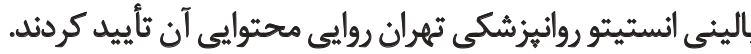

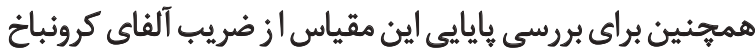

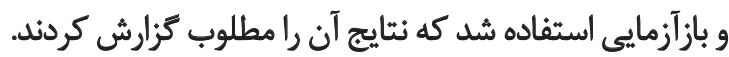

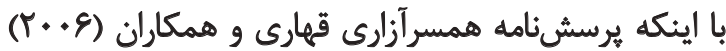

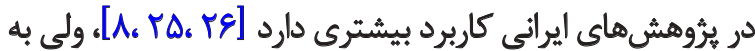

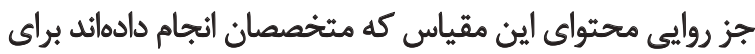
بررسى آن از روشهاي روايى ديكر استفاده نشده است. همجينين

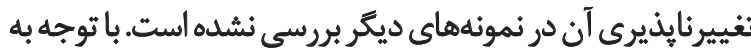

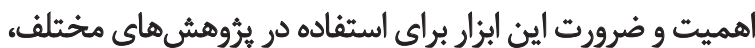

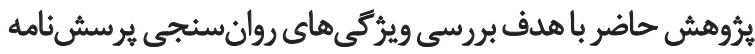

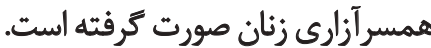

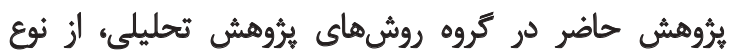

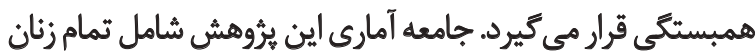

4. Revised Conflict Tactics Scale

5. Abuse Assessment Screen

6. Abusive Behavior Inventory 


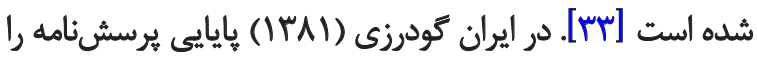

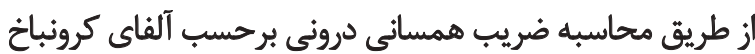

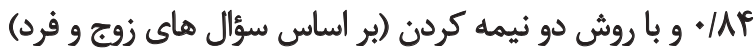

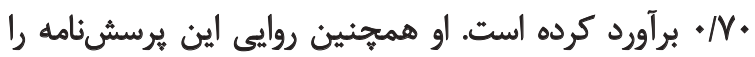

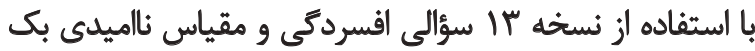

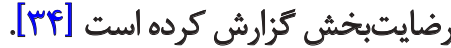

در ثئوهش حاضر براى تحليل دادهها از آزمون تحليل واريانس

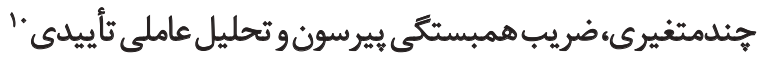

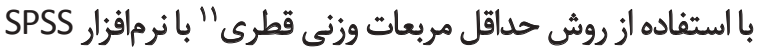

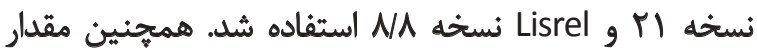

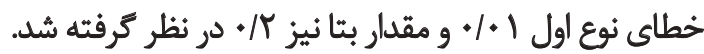

يافتهها

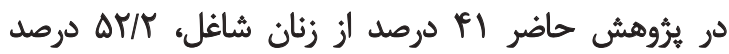

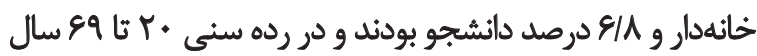

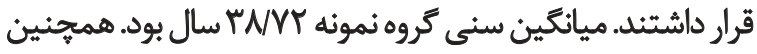

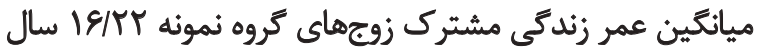

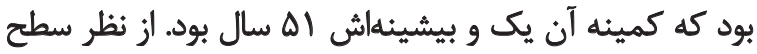

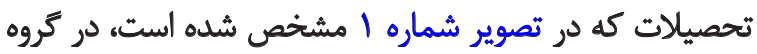

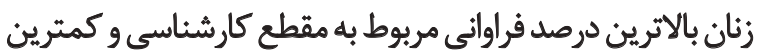

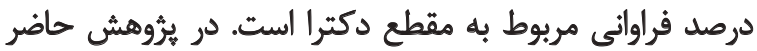

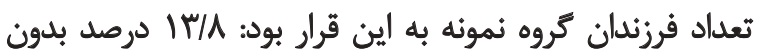

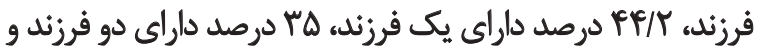

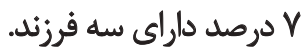

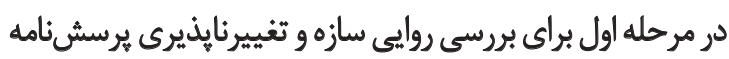

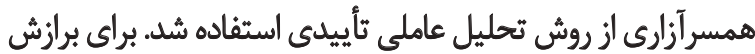

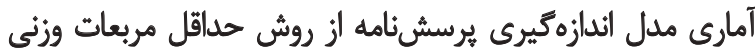

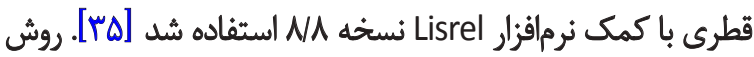

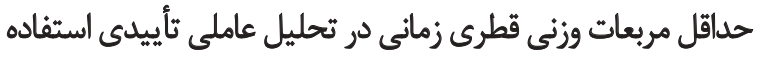

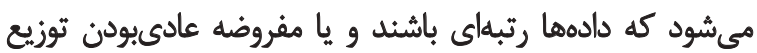

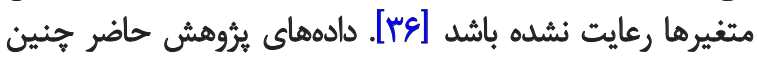

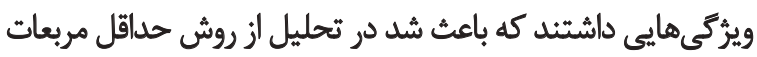

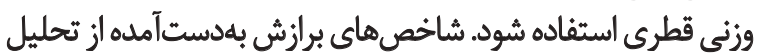

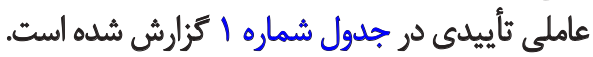

طبق جدول شماره ال از شاخص مجذور كاى دو ساتورا بنتلر

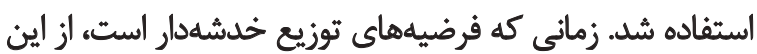

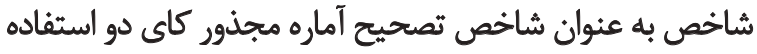

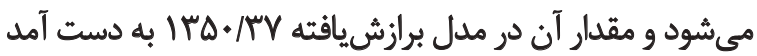

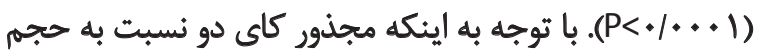

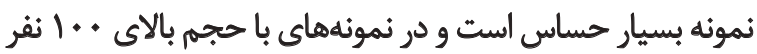

10. Confirmatory Factor Analysis (CFA)

11. Diagonally weighted least squares

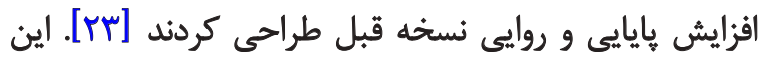

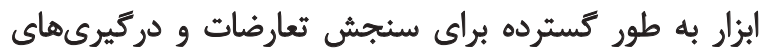

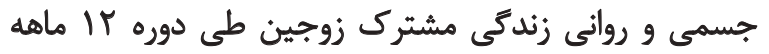

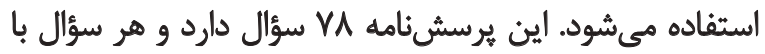

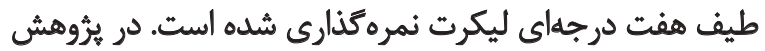

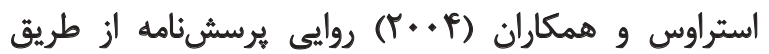

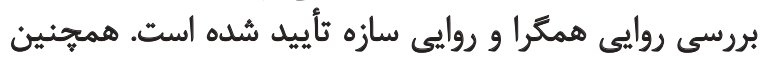

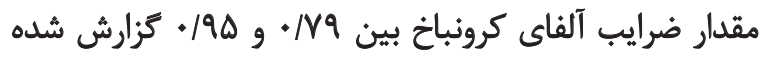

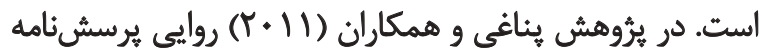

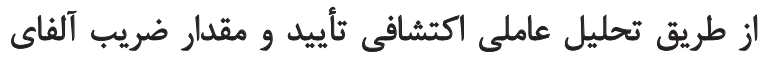

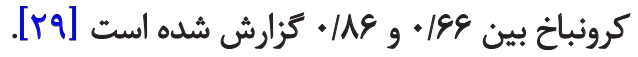

$$
\text { مقياس خودكارآمدى عمومى شرر }
$$

به منظور ارزيابى ميزان خودكارآمدى زنان شركت كنينده در

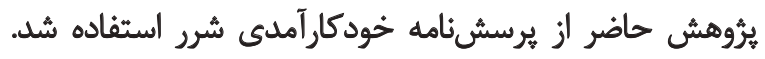

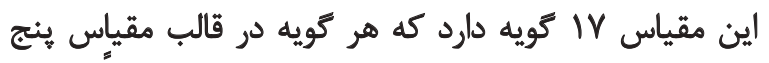

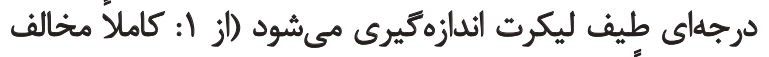

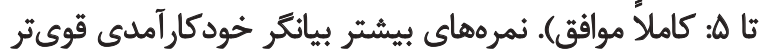

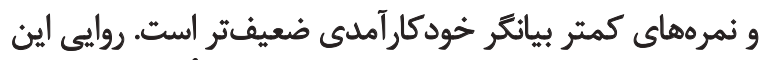

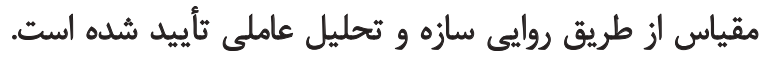

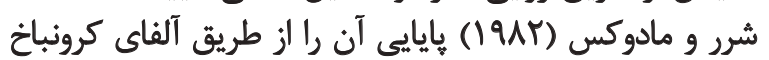

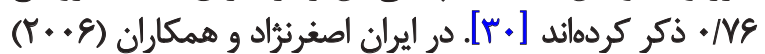

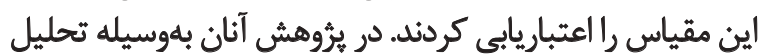

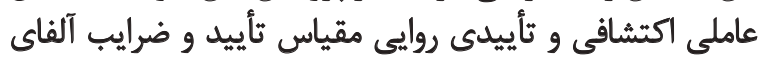
كرونباخ ع 1 • كزارش و شد [أيدى].

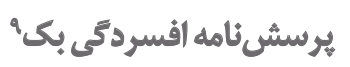

آرون تي بك (1981) به منظور سنجش و ارزيابي وجود

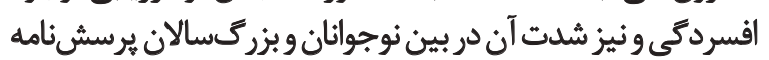

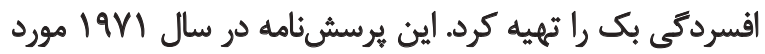

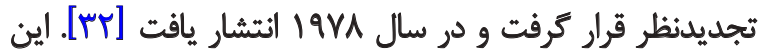

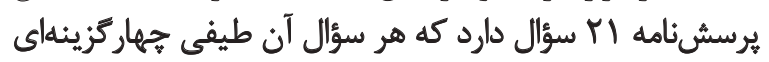

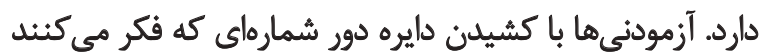

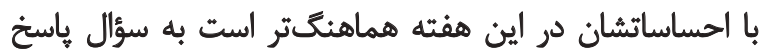

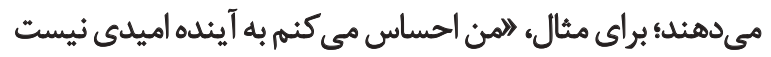

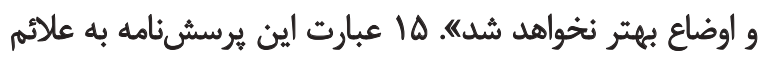

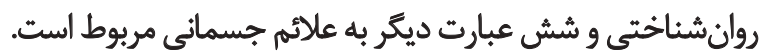

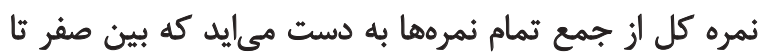

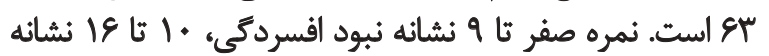

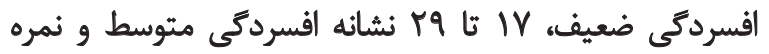

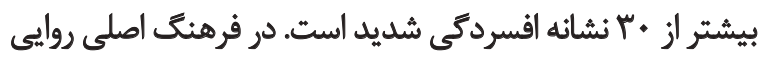

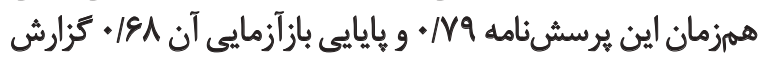

9. Beck Depression Questionnaire 


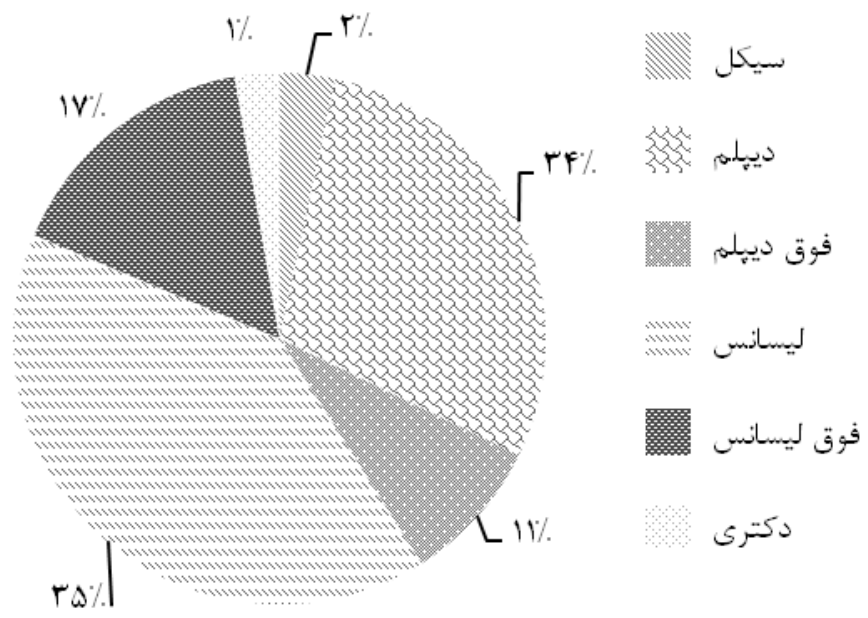

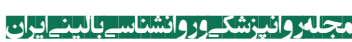

تصوير ا. توزيع فراوانى درصدى بر اساس مقطع تحصيلات كروه زنان

جدول ا. شاخصهاى مطلق، نسبى و ايجازى برازش مدل

\begin{tabular}{|c|c|c|c|c|c|c|c|c|c|}
\hline SRMR $^{7}$ & RMSEA $^{6}$ & $\mathrm{CFF}^{5}$ & NNF/ $1^{4}$ & AGFI $^{3}$ & GFI' ${ }^{2}$ & $\chi^{2} / d f$ & df & ${ }^{1} \mathrm{~S}-\mathrm{Bx}{ }^{2}$ & مدل \\
\hline 1.89 &. .41 & $1 / \cdot \cdot$ & $1 /$. &.$/ 99$ &.$/ 99$ & $1 / \Delta$. & 199 & $\mid r \Delta \cdot / r V$ & مدل اول \\
\hline
\end{tabular}

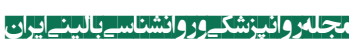

1. Satorra-Bentler chi-square ( $S-B \times 2)$

2. Goodness of Fit Index

3. Adjusted Goodness of Fit Index

4. Non-Normed Fit Index

برازش نشاندهنده قابل قبول بودن مدل است، بنابراين مدل سمعاملى همسرآزارى تأييد مىشود.

نتايج تحليل عاملى تأييدى همراه با شاخصهاى توريفي

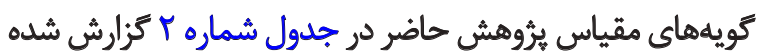

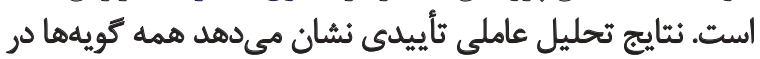

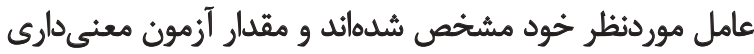

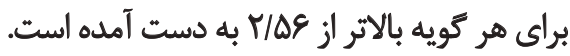

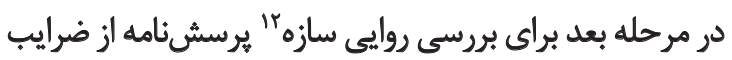

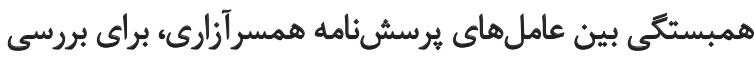

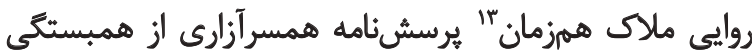

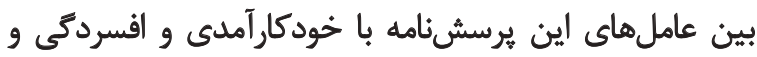

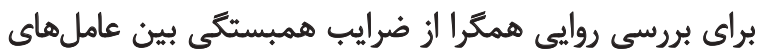

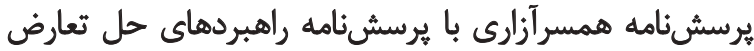

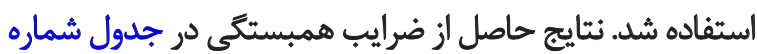

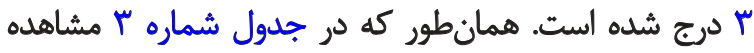

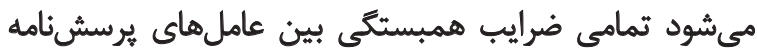

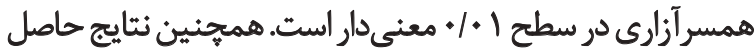

12. Construct validity

13. Concurrent criterion validity
5. Comparative Fit Index

6. Root Mean Square Error of Approximation

7. Standardized Root Mean Square Residual

معنى دار به دست مى آيد، بايد بادرنظركرفتن درجه آزادى تفسير

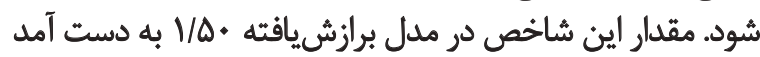
كه اين مقدار از ب كمتر و حاكى از برازش مناسب مدار مدل است. مقدار شاخص ريشه دوم ميانكين مجذورات خطاى ثمان بقريب

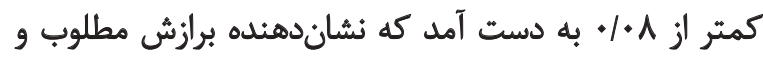

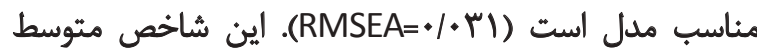

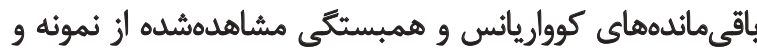

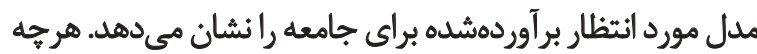

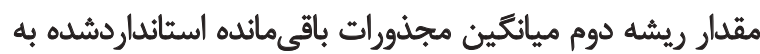

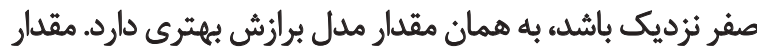

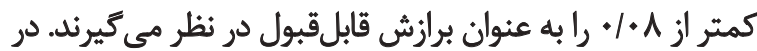

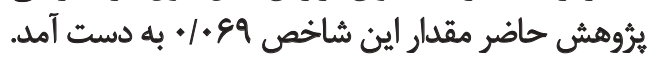

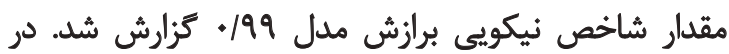

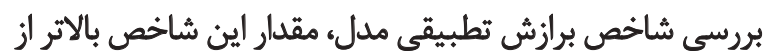

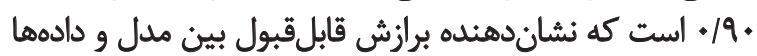

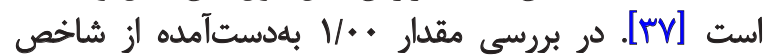

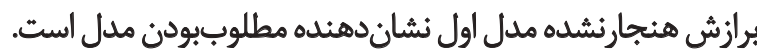

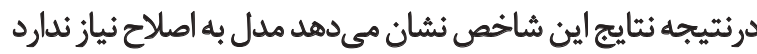

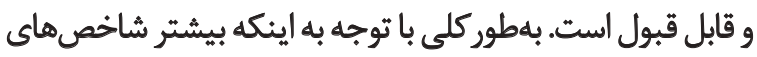


جدول r. نتايج توصيفى و نتايج حاصل از تحليل عاملى كويهماي بريششئامه همسرآزارى عاملها همراه بار عاملى - ماهل

\begin{tabular}{|c|c|c|c|c|c|c|c|}
\hline بدرفتارى & 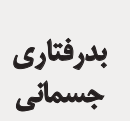 & باطفئى عارى & معيار & معنى أرمون & استاند|راف & ميائكين & كويه \\
\hline & & $\cdot|A|$ & $\%$ & rV/A. & .180 & 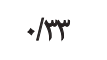 & 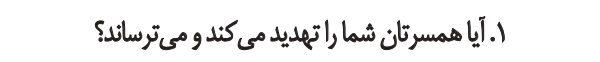 \\
\hline & &.$/ N A$ &.$\%$ & rV/u & .199 & 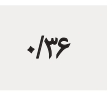 & 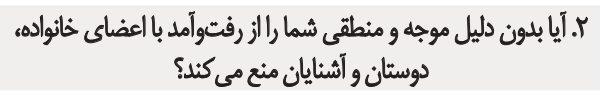 \\
\hline & &.$/ M^{\infty}$ & .1 .9 & IVIVe & $\cdot W$ & . & ". آيا بلون علث خاصى مانع از تحصيل شما مىشود يا شلده است؟ \\
\hline & & $.18 \mathrm{~V}$ & 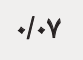 & IV/f. &.$/ 19$ & .10 & 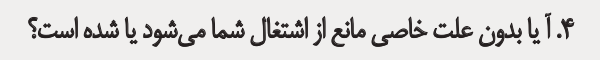 \\
\hline & & ${ }^{\circ} N$ & 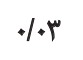 & $r+h q T$ & $\cdot M q$ &.$/ 49$ & هـ آيا رفتارهاى حسادت أميز نسبت به شُما دارد؟ \\
\hline & & $\cdot / \mathrm{NA}$ & $1+4$ & TVITY & $\cdot / M$ &.$|8|$ & 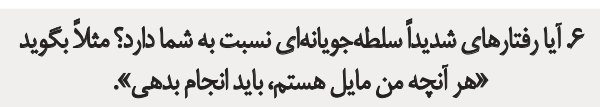 \\
\hline & & $\cdot / A F$ & 1.4 & $W / T_{1}$ & . 14 &.$\pi$ & Y. آيا همسر ثان به قصل تحقير و بى احترامى روى شما اسم كناشته \\
\hline & & $\cdot \mid N$ & . $/ . r$ & me/r & $\cdot M^{e}$ &.$/ P A$ & لر آيا به شما اهائت و توهين هي كند؟ \\
\hline & & .180 & 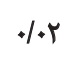 & Is/pr &.$/ M A$ & $\mathrm{~V} \cdot \mathrm{G}$ & ج. آيا الزما ائثقاد هي كند؟ \\
\hline & & $\cdot M$ & $\%$ & TI/TP & . NA & . Me & • أ أيا ملاوم شما را با افراد ديكر مقايسه مي كند؟ \\
\hline & &.$/ M^{\infty}$ &.$/ r^{\prime}$ & (T/Ta & $\cdot M$ & . & 11. آيا موقع اظهارنظر به ذوق شما ميزند؟ \\
\hline & & /Ar & $.1 . r$ & TVRA &.$/ 199$ & . $/ A F$ & rا. آيا به خاطر هر جيزى با شما جروبحث مى كند؟ \\
\hline & & / $/ A Y$ & $\%$ & rapor & $\cdot N$ & זוז/. & سا آيا شما را به باد استيززا مي كيرد؟ \\
\hline & &.$/ M^{\mu}$ & $/ \mu$ & $M / \pi T$ & $\cdot M q$ & .149 & flf آيا شما را به خاطر انجام رفتارهاى ناشايست متهم مى كند؟ \\
\hline & & /AT & $\%$ & $r$ &.$/ 19$ & $\cdot M$ & ها. آيا به خاطر هر جيزى كه ييش مى آيد شما راسرزنش مى كند؟ \\
\hline & &.$/ V E$ & $\%+F$ & $M / 9 \Delta$ &.$/ Q$ & .199 & عا. أيا براى تأمين نيازهاى مادى خود بايد مكرراً الز او خواهش و \\
\hline & &.$/ 9 V$ & 1.4 & Wra &.$/ 9 \%$ &.$M A$ & VIV آيا دير أنجام كارها از مشورت با شما اجتناب هي كند؟ \\
\hline & &.$/ M$ & 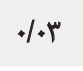 & me/ir & . Af & $\cdot M$ & 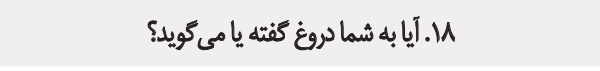 \\
\hline & &. $\operatorname{lep}$ & 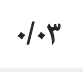 & $18 / \pi$ & $\cdot \| A r$ & Vor & 19. آيا مواردى ييش آمده است كه وعدماي دهد ولى عمل نكند؟ \\
\hline & & IF &.$/ \cdot r$ & WTA & .180 &.$/ 19$ & 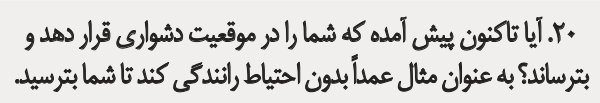 \\
\hline & . & & $\%$ & Pans & .101 &.$/ 19$ & 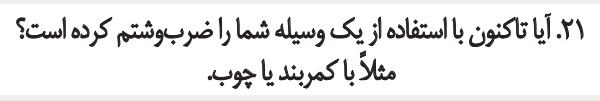 \\
\hline &.$/ 19$ & &.$/ \cdot r$ & TNOY & .18. &.$/ M$ & T ب. آيا به دقعات مشت خور مدايد؟ \\
\hline & $\cdot / A F$ & & 1.4 & $\Pi / \Delta E$ & - AT & - & سٓ. آيا شما را به دفعات هول داده است؟ \\
\hline & $\cdot / 1 / \Delta$ & & $\%$ & $M / 9 P$ & $\cdot / M$ &.$/ W$ & 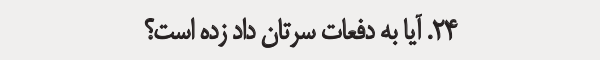 \\
\hline &.$/ 98$ & & / & $N M$ & $\cdot / \mu$ & $\% \Delta$ & هץ. آيا مواردى ييش آمده است كه دست و ياى شما را بسته باشد؟ \\
\hline &.$/ 9 F$ & & 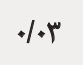 & $\Delta \cdot / M$ &.$/ A 1$ &.$/ r$ & عَ. آيا مواردى ييش آمله است كه بلنتان رازخمى يا كبود كرده باشد؟ \\
\hline
\end{tabular}




\begin{tabular}{|c|c|c|c|c|c|c|c|}
\hline \multicolumn{3}{|c|}{ عاملها همراه بار عاملى } & \multirow[b]{2}{*}{ معيار } & \multirow[b]{2}{*}{ معنى آز مون } & \multirow[b]{2}{*}{ 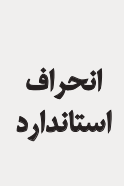 } & \multirow[b]{2}{*}{ 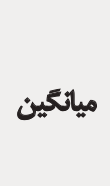 } & \multirow[b]{2}{*}{ كويه } \\
\hline 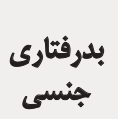 & 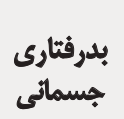 & بل باطفئرى & & & & & \\
\hline & & & 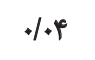 & $M / M$ & 每 & $\cdot / N$ & VI. آيا كتكخُوردن باعث شده است كارتان به درمانكاه بكشد؟ \\
\hline &.$/ 9 \Delta$ & & H.r & $\Delta \Delta / \Delta C$ &.$/ F \Delta$ &.$/ N F$ & 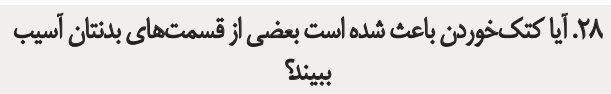 \\
\hline & $\cdot / V^{\prime}$ & & 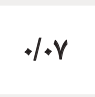 & $19 / 09$ &.$/ M A$ & .1 .8 & 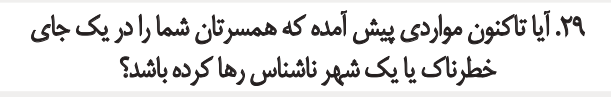 \\
\hline & IAY & & 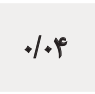 & rqpi & IeV & ת & 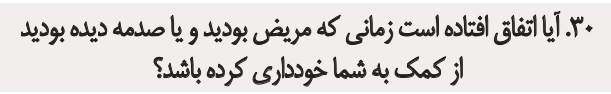 \\
\hline$\cdot|A|$ & & & $+1+f$ & mereq & .18 &.$/ 4 \lambda$ & 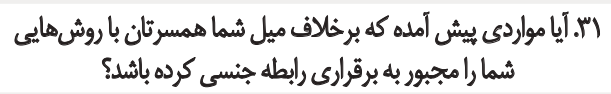 \\
\hline$\cdot / / A$ & & & 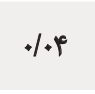 & TVIT & $\cdot M$ & ז"M/. & 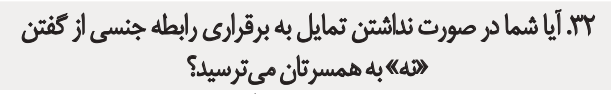 \\
\hline / AF & & & $+1+1$ & $|V / \Psi|$ & .4 &.$M 1$ & سٓر. آيا همسرثان در رابطه جنسى معمولاً عضو جنسى شما راتحقير \\
\hline .188 & & & 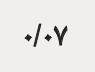 & $I T / M$ &.$(\Delta)$ &.$/ M$ & 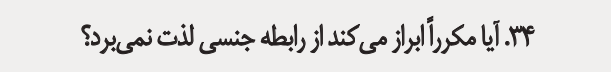 \\
\hline$\cdot|A|$ & & & 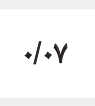 & WTI &.$/ \pi$ &.$/ r$ & هَّ. آيا كلمات زشت و ركيك راهنكام رابطه جنسى با شما به كار \\
\hline .198 & & & $1 \cdot A$ & $W / \Lambda^{*}$. &.$/ 199$ &.$/ N$ & 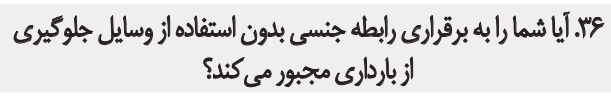 \\
\hline /AY & & &.$/ 19$ & $I Y / A Y$ & سז/ת & .1 .0 & 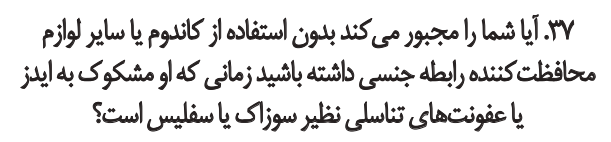 \\
\hline$\cdot / A F$ & & & .1 .8 & $|\sqrt{ }|$ &.$/ N Y$ & .1 .9 & 1" با آيا هنكام رابطه جنسى به شما آسيب رسائده است؟ \\
\hline .119 & & & 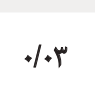 & mepert & / &.$/ M$ & وَّ آيا به دليل خشوئت همسرتان هنكام رابطه جنسي نرد شديلى را \\
\hline$\cdot|A|$ & & & +4 & $W \cdot 1$ & $\cdot / \pi$ & .1 & • +. آيا به دليل خشونت همسرثان هنكام رابطه جنسى دجار خونريزى \\
\hline - MF & & & .1 .8 & WA. & . & .1 .9 & 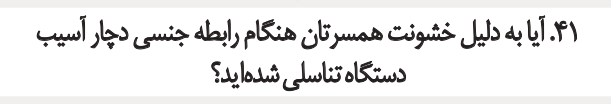 \\
\hline.$/ 19$ & & & $.1 \cdot 0$ & rVAs & . laf &.$/ M$ & Tٓآ آيا از برقرارى رابطه جنسى با همسرتان وحشت داريد؟ \\
\hline$\cdot / M$ & & & .100 & $\mathbb{W} \cdot Y$ & $.1 \Delta \Delta$ &.$/ 4$ & 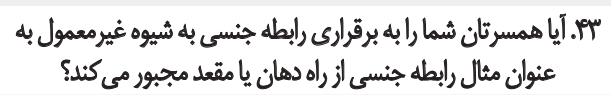 \\
\hline$* 1+\%$ & & & $\pi$ & $\Delta / A$. & $\cdot / \Delta V$ & $\pi$ & 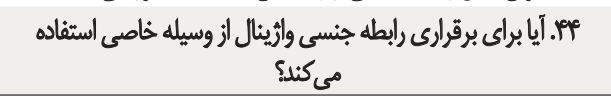 \\
\hline
\end{tabular}

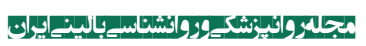

متأهل مثقاضى طلاق بودند و كروه دوم شامل زنان متأهلى بود كه

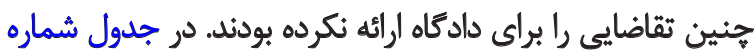

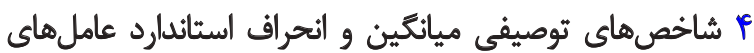

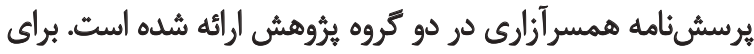

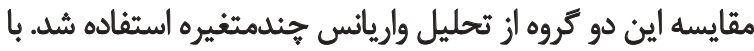

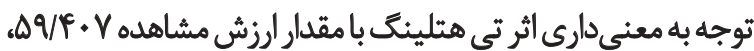

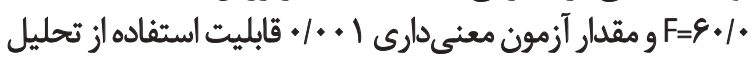

ز ضرايب همبستخى بين عاملهاى يرسشنامه همسرآزارى با

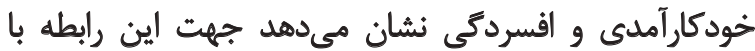

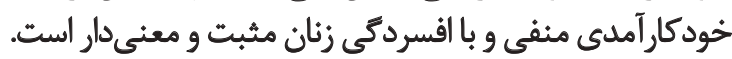

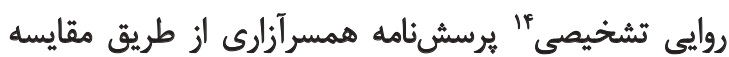

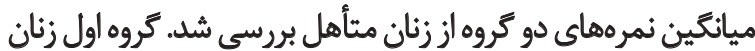

14. Discriminant validity 
جدول r. همبستكى بين عامل هاى يرسشنامه همسرآزارى با راهبردهاى حل تعارض، خودكارآمدى و افسردكى

\begin{tabular}{|c|c|c|c|c|c|}
\hline نمره كل همسرآزارى & بدرفتارى جنسى & بدرفتارى جسمانى & بدرفتارى عاطفى & \multicolumn{2}{|l|}{ مثغيرها } \\
\hline & & & 1 & بدرفتارى عاطفى & 1 \\
\hline & & 1 & $\cdot M T$ & بدرفتارى جسمانى & r \\
\hline & 1 & r & .18 & بدرفتارى جنسى & r \\
\hline 1 &.$N A$ & . IAS &.$/ 90$ & كل يرسش نامه همسرآزازاى & r \\
\hline .18 &.$/ r$ & .109 &.$/ \Delta Y$ & راهبردهاى حل تعارض & $\Delta$ \\
\hline.$- / 41$ &.$- / A T$ &.$- / 49$ &.$- / r q$ & خودكارآمدى & 8 \\
\hline.$/ P \Delta$ &.$/ \pi$ &.$/ 41$ &.$/ 4 \varphi$ & افسردمى & $r$ \\
\hline \multicolumn{2}{|c|}{ 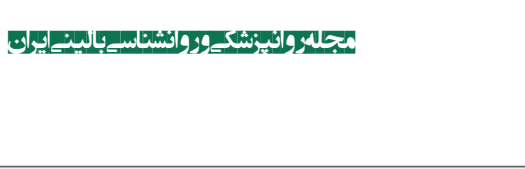 } & \multicolumn{4}{|c|}{ 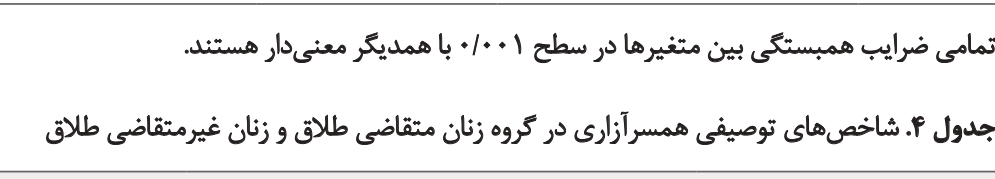 } \\
\hline تعداد نمونه & انحراف استاندارد & مياتكين & ل مكروه زنان & أزارى & \\
\hline $\begin{array}{l}|i| \\
m .\end{array}$ & $\begin{array}{l}\text { ID/FA } \\
\mid K / T .\end{array}$ & 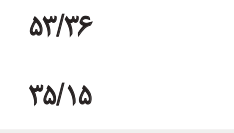 & زنان غنير مثتقاضى طاضلاق طلاق & \multicolumn{2}{|c|}{ بلدفيتارى عاطفى } \\
\hline $\begin{array}{l}|\varphi| \\
m .\end{array}$ & $\begin{array}{l}A n \\
\Delta / 1 .\end{array}$ & $\begin{array}{l}r \cdot \mid q . \\
|r / A|\end{array}$ & زنان زنان مثقاضى طالاق & \multicolumn{2}{|c|}{ بلدرفتارى جسمانى } \\
\hline $\begin{array}{l}\text { ifi } \\
\text { mi. }\end{array}$ & $\begin{array}{l}\text { W/1. } \\
\text { s/q4 }\end{array}$ & $\begin{array}{l}Y M / T Y \\
W / T F\end{array}$ & زنان غنان مثقاضى طلاق & \multicolumn{2}{|c|}{ بلدرفثارى جنسى } \\
\hline
\end{tabular}

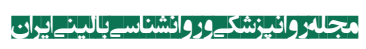

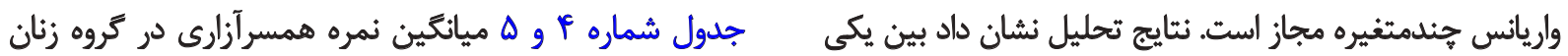

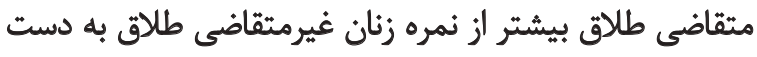
آمد كه اين تفاوت از نظر آمارى معنى دار است.

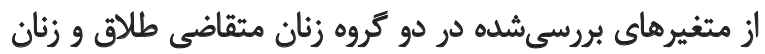
غيرمتقاضى طلاق تفاوت معنى دارى وجود دارد.

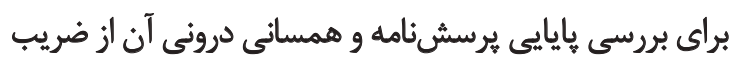

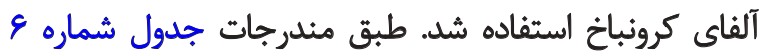

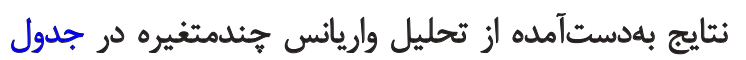

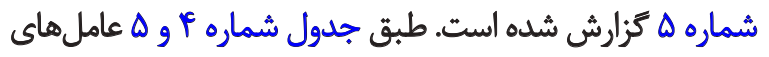

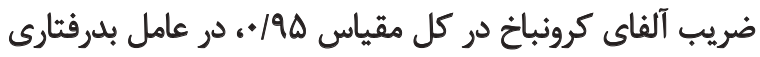

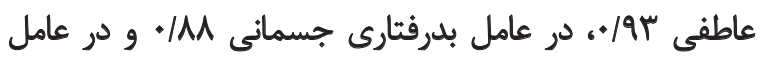

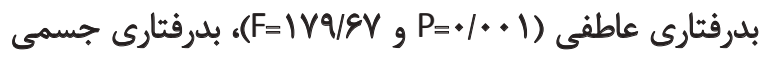

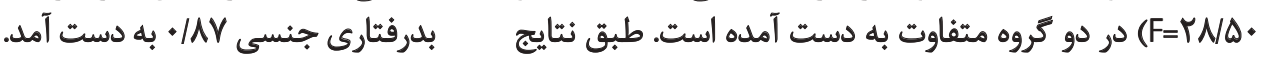

جدول ه. تحليل واريانس جيندمتغيره براى عامل هاى همسرآزارى براى تروه زنان متقاضى طلاق و زنان غيرمتقاضى

\begin{tabular}{|c|c|c|c|c|c|c|}
\hline مجذور اتاي تفكيكي & سطع معني دارى & $\mathbf{F}$ & ميانكّين مجموع مجزورات & ورجه أزادى & هجمع مجذورات & \\
\hline . TNE & $+1+\infty 1$ & IVI/FV & MTIENTY & 1 & MTIENTY & بدرفتارى عاطفى \\
\hline.$/ 194$ & $.1 . .1$ & $1 \cdot V / V 1$ & PFVI/To & 1 & PFVq/FA & بدرفتارى جسمانى \\
\hline 1.9. & .10 .1 & PNTq & $W E / \Delta$. & 1 & $W \& 10$. & بلرقفثارى جنسي \\
\hline
\end{tabular}


جدول و. عاملهاي يرسشنامه همسرآزارى همراه با ضرايب آلفاي كرونباخ

\begin{tabular}{|c|c|c|c|c|}
\hline كل برسش نامه & بدرفتارى جنسى & بدرفتارى جسمانى & بدرفتارى عاطفى & عامل \\
\hline$+/ 9 \Delta$ & $\cdot / A Y$ & $\cdot / M$ & /ar & ضريب آلفاي كرونباخ \\
\hline
\end{tabular}

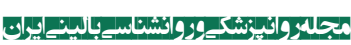

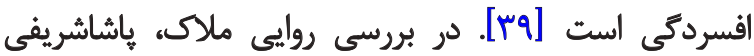

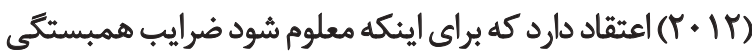

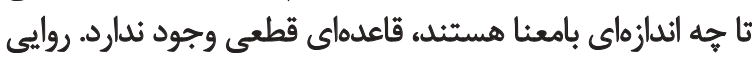

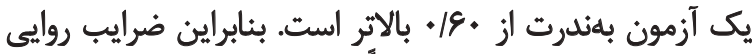

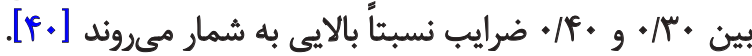

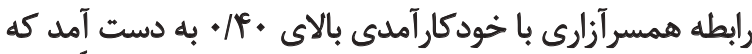

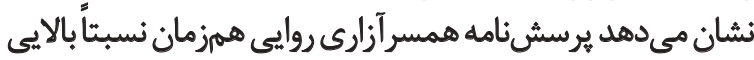
در بررسى عملكرد فردى زنان متأهل دارد.

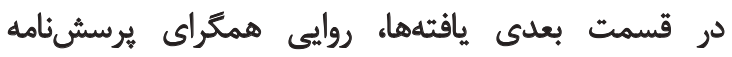

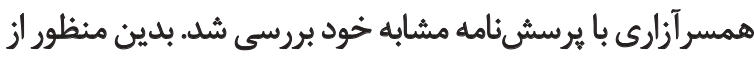

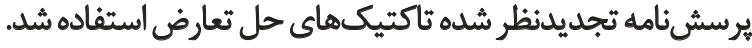

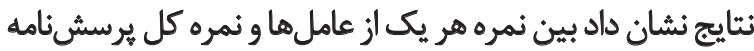

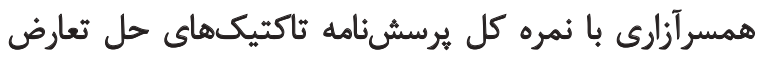

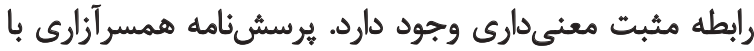

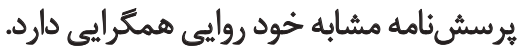

روش ديگرى كه در روايى سازه به كار مىرود برآورد ميزان

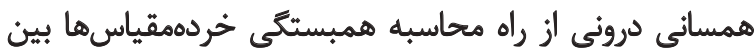

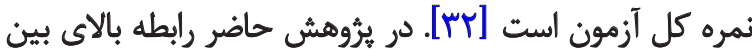

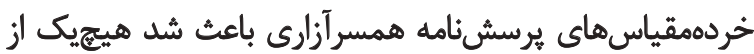

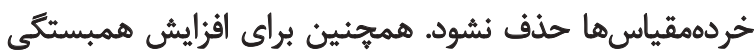

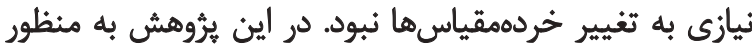

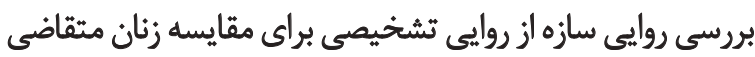

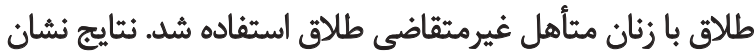

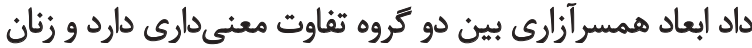

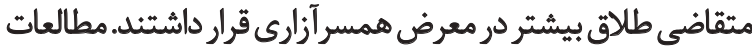

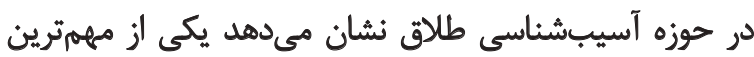

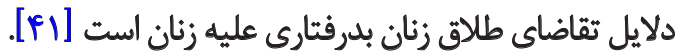
براي بررسي يايايى يرسش نامه از ضريب آلفاي كرونباخ استفاده

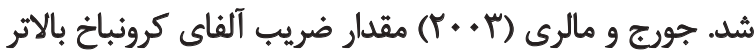

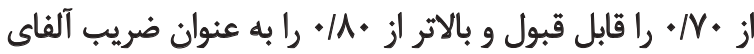

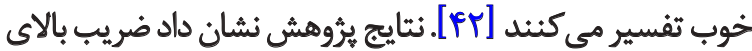

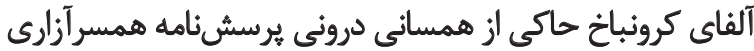

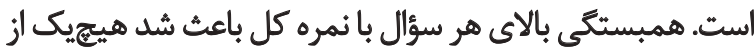
كويdهاى مقياس حذف نشود.

در جمعبندى نهايى بايد بيان كرد كه ضرايب هايايى و

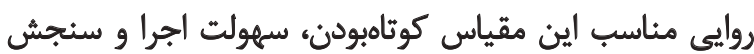

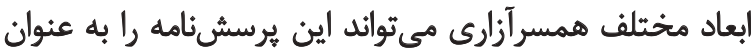

همانطور كه بيشتر اشاره شد يكى از اهداف اين يُروهش

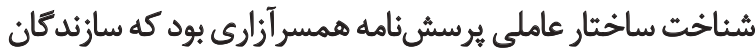

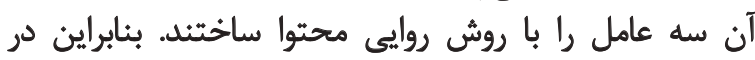

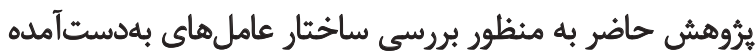

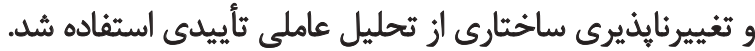

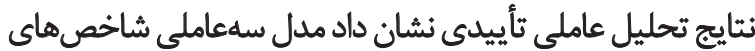

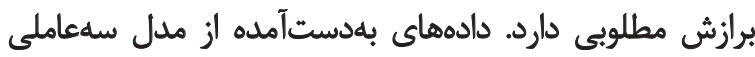

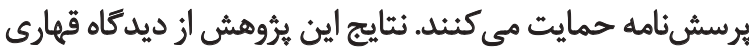

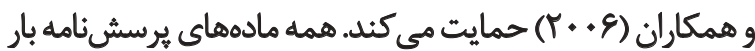

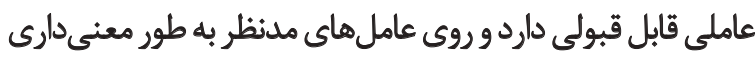

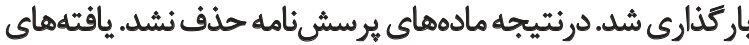

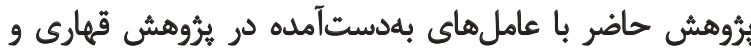

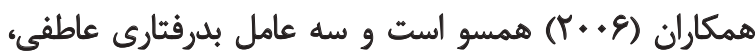

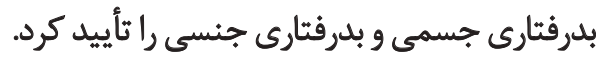

با توجه به اينكه يس از ساخت، اين ابزار در نمونه متفاوت

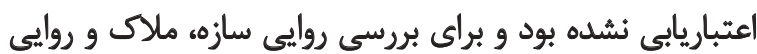

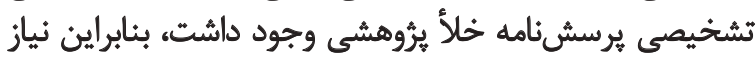

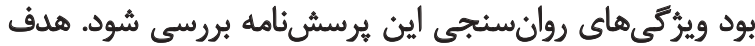

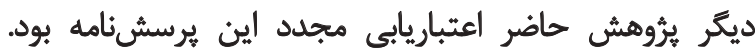

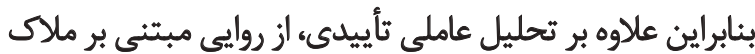

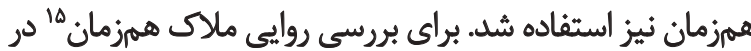

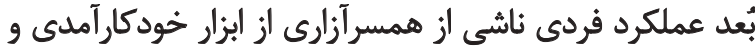

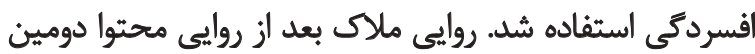

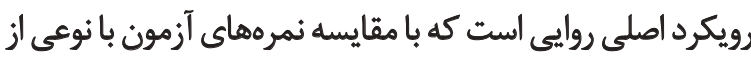

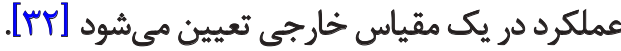

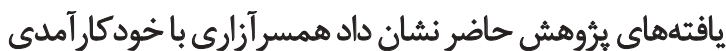

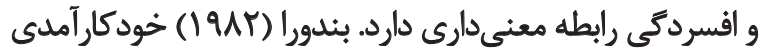

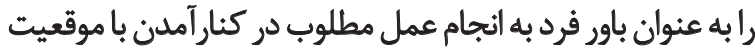

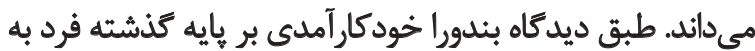

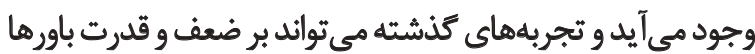

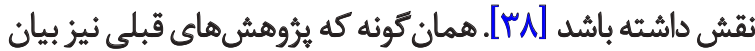

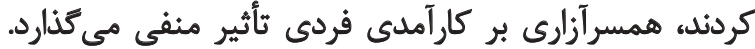

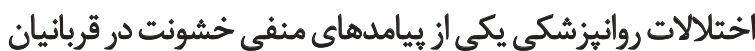

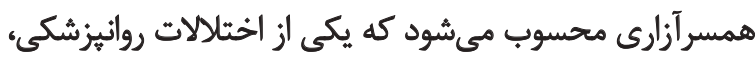

15. Concurrent validity 


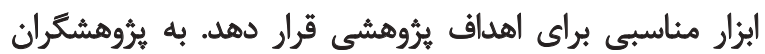

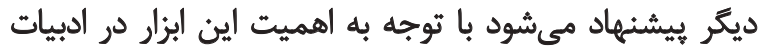

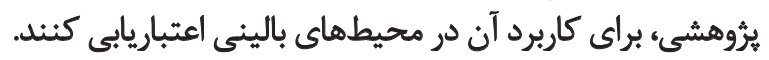

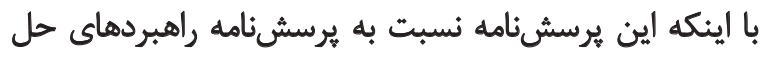

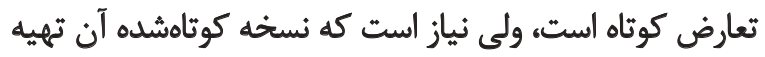

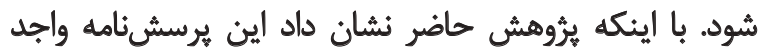

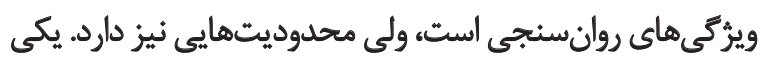

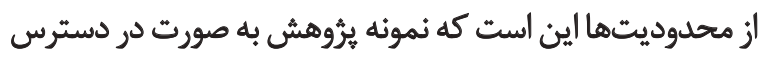

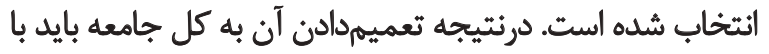

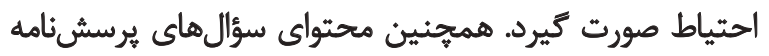

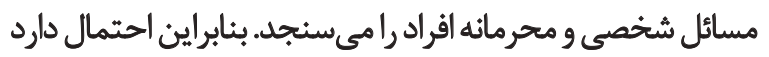

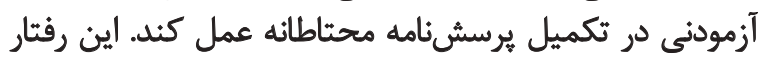

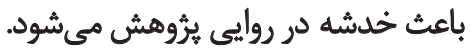
تثيجليرى

در جمعبندى نهايي بايد بيان كرد كه اين يرسشنامه به دليل

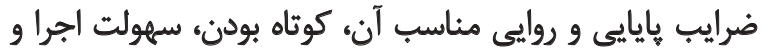

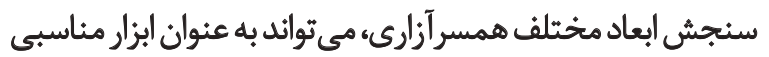

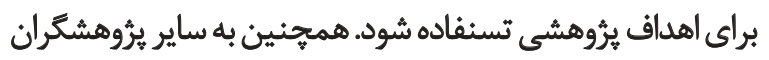

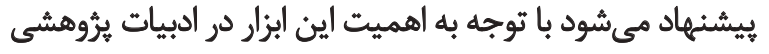

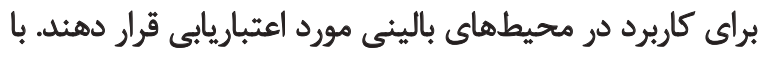

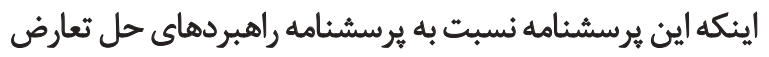

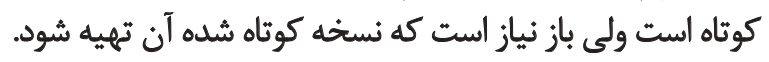

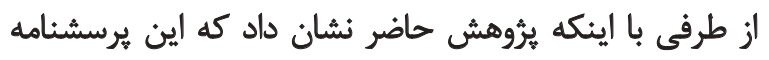

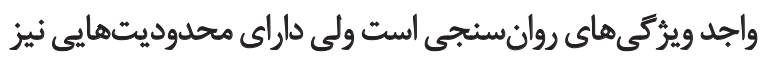

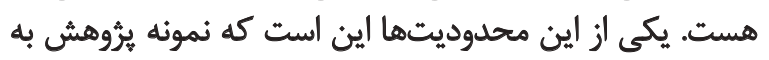

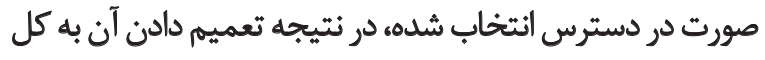

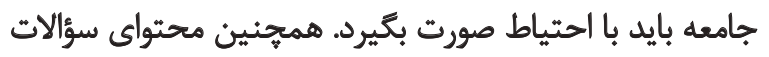

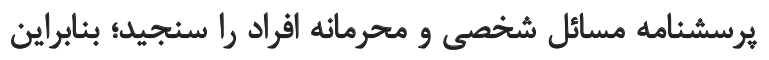

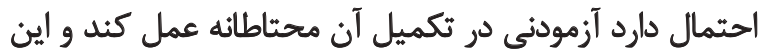
رفتار باعث خدشه در روايى يروهش درئ مي آنود.

$$
\text { سباسكَزارى }
$$

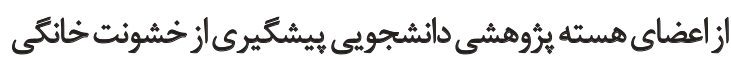

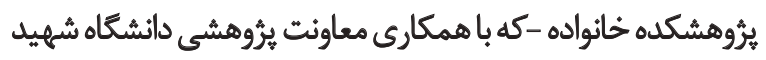

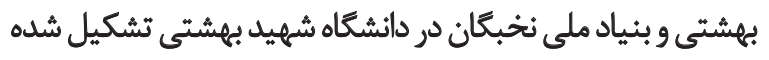

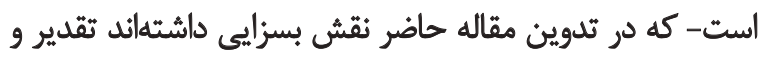

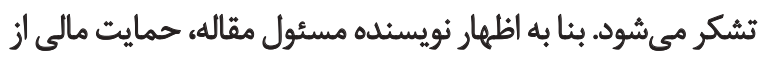
يروهش و تعارض منافع وجود نداشته است. 


\section{References}

[1] Lachs MS, Pillemer K. Elder abuse. The Lancet. 2004; 364(9441):1263-72. doi: 10.1016/s0140-6736(4)17144-4

[2] Krug EG, Mercy JA, Dahlberg LL, Zwi AB. The world report on violence and health. Lancet. 2002; 360(9339):1083-8. doi: 10.1016/ s0140-6736(02)11133-0

[3] Dutton DG. The domestic assault of women: Psychological and criminal justice perspectives. British Columbia: University of British Columbia Press; 2011.

[4] Crowell NA, Burgess AW. Understanding violence against women. Washington, D.C.: American Psychological Association; 1996. doi: $10.1037 / 10204-000$

[5] Richardson J. Identifying domestic violence: cross sectional study in primary care. BMJ. 2002; 324(7332):274. doi: 10.1136/ bmj.324.7332.274

[6] Mulroney J. Australian statistics for domestic violence. Fitzroy North: Australian Domestic \& Family Violence Clearing House: 2003.

[7] Devries KM, Mak JYT, Garcia-Moreno C, Petzold M, Child JC, Falder $\mathrm{G}$, et al. The global prevalence of intimate partner violence against women. Science. 2013; 340(6140):1527-8. doi: 10.1126/science.1240937

[8] Panaghi L, Ghahari S, Ahmadabadi Z, Yoosefi H. [Spouse abuse and mental Health: The role of social support and coping strategies (Persian)]. Journal of Iranian Psychologists. 2008; 5(17):69-79.

[9] Rashti PR, Naderi F. [Descriptive study of domestic violence and its relation with psychological profile of married women (Persian)]. Journal of Women and Culture. 2015; 6(24):51-66.

[10] Ghahari SH, Atef Vahid MK, Yosefi H. [Evaluation of the domestic violence in Azad University of Tonekabon (Persian)] Journal of Mazandaran University of Medical Sciences. 2006; 50(15):83-91.

[11] Shayan A, Masoumi SZ, Kaviani M. [The relationship between wife abuse and mental health in women experiencing domestic violence referred to the forensic medical center of shiraz (Persian)]. Journal of Education and Community Health. 2015; 1(4):517.

[12] Albaugh LM, Nauta MM. Career decision self-efficacy, career barriers, and college women's experiences of intimate partner violence. Journal of Career Assessment. 2005; 13(3):288-306. doi: $10.1177 / 1069072705274958$

[13] Houry D, Kaslow NJ, Thompson MP. Depressive symptoms in women experiencing intimate partner violence. Journal of Interpersonal Violence. 2005; 20(11):1467-77. doi: $10.1177 / 0886260505278529$

[14] Afifi TO, Henriksen CA, Asmundson GJG, Sareen J. Victimization and perpetration of intimate partner violence and substance use disorders in a nationally representative sample. The Journal of Nervous and Mental Disease. 2012; 200(8):684-91. doi: 10.1097/ nmd.0b013e3182613f64

[15] Van Hasselt VB, Morrison RL, Bellack AS, Hersen M. Handbook of family violence. New York: Springer; 1988. doi: 10.1007/978-14757-5360-8
[16] Devries KM, Mak JY, Bacchus LJ, Child JC, Falder G, Petzold $\mathrm{M}$, et al. Intimate partner violence and incident depressive symptoms and suicide attempts: A systematic review of longitudinal studies. PLoS Medicine. 2013; 10(5):1001439. doi: 10.1371/journal. pmed.1001439

[17] Hekmat Kh, Mortazavi E, Haghighi MH, Farzad Hoseini S. [Survey of prevalence and severity of suicidal ideation in women with spouse abuse referred to Ahvaz Forensic Medicine Center in 2013 (Persian)]. Journal of Zabol University of Medical Sciences and Health Services. 2016; 7(4):1-11.

[18] Rasulian M, Bolhari J, Nojomi M, Habib S, Mirzaei Khoshalani M. [Theories and interventional models of intimate partner violence: Suggesting an interventional model based on primary health care system in Iran (Persian)]. Iranian Journal of Psychiatry and Clinical Psychology. 2015; 21(1):3-16.

[19] McFarlane J, Hughes RB, Nosek MA, Groff JY, Swedlend N, Dolan Mullen P. Abuse assessment screen-disability (AASD): Measuring frequency, type, and perpetrator of abuse toward women with physical disabilities. Journal of Women's Health \& Gender-Based Medicine. 2001; 10(9):861-6. doi $10.1089 / 152460901753285750$

[20] Shepard MF, Campbell JA. The abusive behavior inventory. Journal of Interpersonal Violence. 1992; 7(3):291-305. doi $10.1177 / 088626092007003001$

[21] Straus MA. Measuring intrafamily conflict and violence: The Conflict Tactics (CT) scales. Journal of Marriage and the Family. 1979; 41(1):75-88. doi: 10.2307/351733

[22] Straus MA. The conflict tactics scales and its critics: An evaluation and new data on validity and reliability. Durham: University of New Hampshire Pub; 1987

[23] Straus MA, Hamby SL, Boney-McCoy S, Sugarman DB. The revised Conflict Tactics Scales (CTS2). Journal of Family Issues. 1996; 17(3):283-316. doi: 10.1177/019251396017003001

[24] Panaghi L, Dehghani M, Abbasi M, Mohammadi S, Maleki G. [Investigating reliability, validity and factor structure of the revised conflict tactics scale (Persian)]. Journal of Family Research 2011; 7(1):103-7.

[25] Panaghi L, Pirouzi D, Shirinbayan M, Ahmadabadi Z. [The role of personality and demographic traits in spouse abuse (Persian)] Iranian Journal of Psychiatry and Clinical Psychology. 2011; 17(2):126-35.

[26] Navroi S, Omid S, Zeinali S, Khasteganan N. [Relationship between spouse abuse, social support and perceived stress in women with addicted and non-addicted husbands in Rasht city (Persian)]. Journal of Holistic Nursing and Midwifery. 2012; 22(2):25-32.

[27] Hooman H. [Structural equation modeling using Lisrel software (Tehran)]. Tehran: SAMT; 2012

[28] Boomsma A, Hoogland JJ. The robustness of Lisrel modeling revisited. In: Cudeck R, du Toit S, Sorbom D. Structural Equation Models: Present and Future. Lincolnwood: Scientific Software International; 2001.

[29] Straus MA. Cross-cultural reliability and validity of the revised conflict tactics scales: A study of university student dating couples in 17 nations. Cross-Cultural Research. 2004; 38(4):407-32. doi: $10.1177 / 1069397104269543$ 
[30] Sherer M, Maddux JE, Mercandante B, Prentice-Dunn S, Jacobs B, Rogers RW. The self-efficacy scale: Construction and validation. Psychological Reports. 1982; 51(2):663-71. doi: 10.2466/ pr0.1982.51.2.663

[31] Asghar Nejad T, Ahmadi M, Farzad V, Khodapanahi M K. [The psychometric properties of the scale efficacy study (Persian)]. Journal of Psychology. 2006; 3(10); 262-274.

[32] Groth-Marnat G. Handbook of psychological assessment. New Jersi: John Wiley \& Sons; 2009.

[33] Beck AT, Steer RA, Carbin MG. Psychometric properties of the Beck Depression Inventory: Twenty-five years of evaluation. Clinical Psychology Review. 1988; 8(1):77-100. doi: 10.1016/02727358(88)90050-5

[34] Goudarzi MA. [The validity and reliability of the Beck Hopelessness Scale in a group of students from Shiraz University (Persian)]. Journal of Social Sciences and Humanities of Shiraz University. 2008; 2(18):26-39.

[35] Jöreskog KG, So"rbom D. Lisrel 8.7 for Windows [Computer Software]. Lincolnwood: Scientific Software International, Inc; 2004.

[36] Li CH. Confirmatory factor analysis with ordinal data: Comparing robust maximum likelihood and diagonally weighted least squares. Behavior Research Methods. 2015; 48(3):936-49. doi: 10.3758/s13428-015-0619-7

[37] Meyers LS, Gamst G, Guarino AJ. Applied multivariate research: Design and interpretation. California: Sage Publications; 2006.

[38] Bandura A. Self-efficacy mechanism in human agency. American Psychologist. 1982; 37(2):122-47. doi: 10.1037/0003066x.37.2.122

[39] Iverson KM, Gradus JL, Resick PA, Suvak MK, Smith KF, Monson CM. Cognitive-behavioral therapy for PTSD and depression symptoms reduces risk for future intimate partner violence among interpersonal trauma survivors. Journal of Consulting and Clinical Psychology. 2011; 79(2):193-202. doi: 10.1037/a0022512

[40] Pashasharifi H. Principles of psychometric and psychological testing (Persian)]. Tehran: Roshd; 2012.

[41] Sadrolashrafi M, Khonakdar M, Shamkhani A, Yusefi Afrashteh M. [Pathology of divorce (factors and causes) and solutions to prevent (Persian)]. Journal of Cultural Engineering. 2011; $7(73) ; 26-53$.

[42] George D. SPSS for windows step by step: A simple study guide and reference, 17.0 update, 10/e. Noida: Pearson Education India; 2011. 